\title{
The Blood Flow Shutdown Induced by Combretastatin A4 Impairs Gemcitabine Delivery in a Mouse Hepatocarcinoma
}

\author{
Anne-Catherine Fruytier ${ }^{1}$, Cecile S. Le Duff ${ }^{2}$, Chrystelle Po ${ }^{1}$, Julie Magat ${ }^{1}$, \\ Caroline Bouzin ${ }^{3}$, Marie-Aline Neveu ${ }^{1}$, Olivier Feron ${ }^{3}$, Benedicte F. Jordan ${ }^{1}$ and \\ Bernard Gallez ${ }^{1 *}$
}

${ }^{1}$ Biomedical Magnetic Resonance Research Group, Louvain Drug Research Institute, Université Catholique de Louvain, Brussels, Belgium, ${ }^{2}$ Institute of Condensed Matter and Nanosciences, Université Catholique de Louvain, Louvain-la-Neuve, Belgium, ${ }^{3}$ Institut de Recherche Expérimentale et Clinique, Pole of Pharmacology, Angiogenesis and Cancer Research Laboratory, Université Catholique de Louvain, Brussels, Belgium

\section{OPEN ACCESS}

Edited by:

Agnès Noël,

University of Liège, Belgium

Reviewed by:

Andrea Lapucci,

University of Florence, Italy

Nicolas Etique,

University of Reims

Champagne-Ardenne, France

Christian Stockmann

French Institute of Health and Medical

Research, France

*Correspondence:

Bernard Gallez

bernard.gallez@uclouvain.be

Specialty section

This article was submitted to Pharmacology of Anti-Cancer Drugs,

a section of the journal

Frontiers in Pharmacology

Received: 08 June 2016 Accepted: 07 December 2016 Published: 23 December 2016

Citation:

Fruytier A-C, Le Duff CS, Po C

Magat J, Bouzin C, Neveu M-A Feron O, Jordan BF and Gallez B (2016) The Blood Flow Shutdown

Induced by Combretastatin A4 Impairs Gemcitabine Delivery in a

Mouse Hepatocarcinoma

Front. Pharmacol. 7:506

doi: 10.3389/fphar.2016.00506
In recent clinical studies, vascular disrupting agents (VDAs) are mainly used in combination with chemotherapy. However, an often overlooked concern in treatment combination is the VDA-induced impairment of chemotherapy distribution in the tumor. The work presented here investigated the impact of blood flow shutdown induced by Combretastatin A4 (CA4) on gemcitabine uptake into mouse hepatocarcinoma. At $2 \mathrm{~h}$ after CA4 treatment, using DCE-MRI, a significant decrease in the perfusion-relevant parameters $K^{\text {trans }}$ and $\mathrm{Vp}$ were observed in treated group compared with the control group. The blood flow shutdown was indeed confirmed by a histology study. In a third experiment, the total gemcitabine uptake was found to be significantly lower in treated tumors, as assessed in a separate experiment using ex vivo fluorine nuclear magnetic resonance spectroscopy. The amount of active metabolite gemcitabine triphosphate was also lower in treated tumors. In conclusion, the blood flow shutdown induced by VDAs can impact negatively on the delivery of small cytotoxic agents in tumors. The present study outlines the importance of monitoring the tumor vascular function when designing drug combinations.

Keywords: ${ }^{19} \mathrm{~F}$ NMR, tumor perfusion, DCE-MRI, vascular disrupting agent, drug delivery

\section{INTRODUCTION}

Tumor vasculature is an attractive target in anticancer therapy because of its critical role in tumor growth, development, and metastasis (Folkman, 1995). Moreover, the unique characteristics of tumor vasculature compared with that of normal tissue can be selectively exploited by antivascular therapies. Unlike antiangiogenic agents, which prevent the development of new blood vessels, vascular disrupting agents (VDAs) target the established tumor vasculature causing a rapid collapse in tumor blood flow leading to secondary extensive tumor necrosis (Thorpe, 2004; Tozer et al., 2005). Several preclinical studies have shown that VDAs induce necrosis in the poorly perfused core regions but spare tumor cells in periphery (Blakey et al., 2002; Chaplin and Hill, 2002; Siemann, 2011). This "viable rim" forms the rationale for combination approaches since VDAs affect the central part of the tumor that is often resistant to conventional therapies, whereas conventional 
treatments are more active on highly proliferating and well oxygenated peripheral cells (Tozer et al., 2008). Consequently, VDAs are mainly used in combination with chemotherapy in current clinical studies.

However, an often overlooked concern is the possible VDAinduced impairment of drug distribution in the tumor (Cesca et al., 2013). Indeed, currently, combination strategies are often empirical whereas a careful sequence and schedule of administration of treatments are needed to avoid negative interactions and to potentiate synergic efficacy of the drugs (Wang et al., 2012; Cesca et al., 2013). In this context, early biomarkers of response are needed to assess the impact of VDA on chemotherapy delivery and to determine optimal timing of administration in early clinical trials. Dynamic contrastenhanced magnetic resonance imaging (DCE-MRI) is the imaging method of choice to assess early microvascular changes induced by antivascular treatments (O'Connor et al., 2012). It generates non-invasive maps of hemodynamics parameters such as blood flow and/or vascular permeability. Moreover, it has been suggested that DCE-MRI with low molecular weight gadoliniumbased contrast agents can be used to predict the delivery of drugs with similar size to the interstitium of solid tumors (Artemov et al., 2001).

The first aim of the present work was to investigate how a negative modulation of tumor blood flow could affect the uptake of chemotherapeutic agents into the tumor. A second objective was to appraise the potential of DCE-MRI to predict the impact of the modulation of tumor perfusion on chemotherapy delivery. To that purpose, we first monitored the early effects of Combretastatin A4 (CA4), the lead compound of VDAs, using DCE-MRI. CA4 acts as a tubulin-binding agent, leading to a destabilization of the tubulin polymers of the cytoskeleton of proliferating endothelial cells. This way, an acute increase in tumor vascular permeability is induced, which in turn triggers several changes that together, decrease blood flow (Tozer et al., 2005). The perfusion parameters $\mathrm{K}^{\text {trans }}$ (the volume transfer constant between blood plasma and extravascular extracellular space) and Vp (the blood plasma volume fraction) were determined since it has been previously shown to be relevant markers of CA4 efficacy (Maxwell et al., 2002; Nielsen et al., 2010). To corroborate MRI findings, a qualitative histology study was conducted in a separate group of animals to assess the perfusion using the Hoescht 33342 marker. In parallel, the uptake of the fluorinated chemotherapeutic agent gemcitabine was assessed ex vivo using fluorine nuclear magnetic resonance spectroscopy ( ${ }^{19}$ F NMR).

\section{MATERIALS AND METHODS}

\section{Animals and Tumor Model}

Transplantable liver tumors (TLT hepatocarcinoma Taper et al., 1966) were induced i.m. into the right gastrocnemius muscle of 5-week-old male NMRI mice (Janvier, France). Tumors were allowed to reach up to $8 \pm 0.5 \mathrm{~mm}$ in diameter prior to experimentation. For all experiments, mice were anesthetized using isoflurane (3\% for induction, $1.5 \%$ for maintenance, mixed with air). Body temperature was maintained at $37.0 \pm 1.0^{\circ} \mathrm{C}$ with a circulating water blanket and monitored together with respiration rate during experiments. All animal experiments were performed in accordance to national animal care regulations with the approval of local Ethics Board 2010/UCL/MD/01. CA4 (Sigma-Aldrich, Belgium) dissolved in DMSO was delivered i.p. at a dose of $100 \mathrm{mg} / \mathrm{kg}$ (Grosios et al., 1999).

${ }^{19} \mathrm{~F}$ NMR and DCE-MRI experiments were performed on separate cohorts of mice, because of the possible influence of the contrast agent on fluorine relaxation times (Ratner et al., 1989).

\section{DCE-MRI}

Mice were divided into an untreated control group receiving vehicle (DMSO) $(n=6)$ and another treated group receiving CA4 $(n=6)$. DCE-MRI acquisition was carried out $2 \mathrm{~h}$ after treatment, a timing for which we anticipated an important reduction in tumor perfusion (Thorpe, 2004). The contrast agent (CtAg) used was gadoterate meglumine, a small gadolinium chelate routinely used in clinics $(0.286 \mathrm{mmol} \mathrm{Gd} / \mathrm{kg})$. A $24 \mathrm{G}$ catheter was inserted in the caudal vein of mice for CtAg injection.

\section{Acquisition}

A quadrature whole body coil was used for radiofrequency transmission and reception. High-resolution multi-slice T2weighted spin echo anatomical imaging was performed just before DCE-MRI. For DCE-MRI, T1 weighted gradient echo images were obtained with a fast low angle shot sequence with the following parameters: repetition time $=15 \mathrm{~ms}$, echo time $=2.074$ ms, flip angle $=40^{\circ}$, matrix $=128 \times 64$, field of view $=40 \times$ $40 \mathrm{~mm}$, zero-fill acceleration factor $=1.4$. A first set of $400 \mathrm{scans}$ with a temporal resolution of $1.19 \mathrm{~s}$ was acquired, with CtAg manually administered intravenously after the twentieth scan over 2 s. Afterwards, a slower DCE data set was acquired with a temporal resolution of $10.1 \mathrm{~s}$ to monitor the CA washout (300 images). A proton density weighted image was acquired before T1-weighted sequences with the following parameters: repetition time $=3500 \mathrm{~ms}$, echo time $=2.074 \mathrm{~ms}$, flip angle $=40^{\circ}$, matrix $=128 \times 64$, field of view $=40 \times 40 \mathrm{~mm}$.

\section{Data Analysis}

DCE-MRI data were analyzed using the extended Tofts model (ETM). A population-averaged arterial input function was used, previously obtained in iliac artery/vein of the same mouse model (Fruytier et al., 2014a). A global region of interest (ROI) was manually delineated to cover the entire tumor area (using the T2-weighted anatomical images as reference).

The signal intensity obtained from the FLASH sequence is (Buckley and Parker, 2005):

$$
S=S_{0} \frac{\sin \alpha \cdot\left(1-\exp ^{\frac{-T R}{T_{1}}}\right)}{\left(1-\cos \alpha \cdot \exp ^{\frac{-T R}{T_{1}}}\right)} \cdot \exp \left(-\frac{T E}{T_{2}{ }^{*}}\right),
$$

where $S_{0}$ is a scaling factor comprising proton density and instrumental factors and is determined by the proton density sequence, $\alpha$ is the flip angle, TR is the repetition time, and TE is the echo time. Signal dependence on $T_{2}{ }^{*}$ was neglected due to 
the short echo time ( $T E=2.074 \mathrm{~ms})$. In tumors, the relationship between relaxation rate $\left(1 / T_{1}\right)$ and $\mathrm{CA}$ concentration can be predicted by Buckley and Parker (2005):

$$
\frac{1}{T_{1}}=\frac{1}{T_{10}}+r_{1} \cdot C(t),
$$

where $r_{1}$ is the longitudinal relaxivity of the CA at $11.7 \mathrm{~T}, T_{10}$ is the longitudinal relaxation time in the absence of the CA, and $C(t)$ is the concentration of CA in tissue.

The measured tumor concentration time course $\left[C_{t}(t)\right]$ of pixels was individually fitted to the ETM using a LevenbergMarquardt nonlinear least-squares procedure:

$$
\begin{aligned}
C_{t}(t)= & K^{\text {trans }} \cdot D \cdot \sum_{i=1}^{2} a_{i} \frac{\exp \left(-k_{e p} t\right)-\exp \left(-m_{i} t\right)}{m_{i}-k_{e p}} \\
& +v_{p} \cdot D \cdot \sum_{i=1}^{2} a_{i} \cdot \exp \left(-m_{i} t\right)
\end{aligned}
$$

where $\mathrm{K}^{\text {trans }}$ is the volume transfer constant between blood plasma and extravascular extracellular space (EES) $\left[\mathrm{min}^{-1}\right], v_{p}$ is the blood plasma volume per unit volume of tissue, and $k_{e p}$ is the rate constant between EES and blood plasma [ $\mathrm{min}^{-1}$ ] (Tofts et al., 1999). $\mathrm{D}$ is the CA bolus dose. The constants $a_{i}$ and $m_{i}$ are population-averaged mean amplitudes and decay rates obtained previously in same tumor model: $a_{1}=16.41 ; m_{1}=11.20 ; a_{2}=$ $8.41 ; m_{2}=0.27$ (Fruytier et al., 2014a). Some pixels provide nonphysiological values of $K^{\text {trans }}\left(K^{\text {trans }}<0, K^{\text {trans }}>1 \mathrm{~min}^{-1}\right)$ and/or $v_{p}\left(v_{p}<0, v_{p}>1\right)$. For mean calculations, these pixels were set to zero or 1 respectively.

\section{Ex vivo ${ }^{19} \mathrm{~F}$ NMR}

All mice received gemcitabine ( $800 \mathrm{mg} / \mathrm{kg}$, IP injection, Hospira) $2 \mathrm{~h}$ after vehicle $(n=6)$ or CA4 treatment $(n=6)$. Tumors were carefully excised $2 \mathrm{~h}$ after gemcitabine treatment and snap-frozen for ex vivo ${ }^{19} \mathrm{~F}$ NMR experiments.

\section{Tissue Extraction}

The following protocol was adapted from Refs (Olive et al., 2009; Bapiro et al., 2011). The snap-frozen tumors were weighted and homogenized in four volumes of ice-cold acetonitrile for $5 \mathrm{~min}$. An equal volume of ice-cold water was added and homogenization was carried on for a further $5 \mathrm{~min}$. Samples were incubated on ice for $10 \mathrm{~min}$ before being centrifuged at $14,000 \mathrm{~g}$ for $10 \mathrm{~min}$ at $4^{\circ} \mathrm{C}$. The supernatants were transferred to vials and stored at $-80^{\circ} \mathrm{C}$ before lyophilization.

\section{Acquisition}

The Freeze-dried tumor supernatants were re-suspended in $500 \mu \mathrm{l}$ of deuterium oxide $\left(\mathrm{D}_{2} 0\right)$, spiked with 185 nmoles of 2-Fluoro-2'-deoxyadenosine (2F2dA), used as the external standard.

${ }^{19}$ F NMR measurements were carried out on a Bruker Avance II NMR spectrometer operating at $300 \mathrm{MHz}$ for ${ }^{1} \mathrm{H}$ and fitted with a $5 \mathrm{~mm}$ BBFO probe. $1 \mathrm{D}{ }^{19} \mathrm{~F}$ spectra were acquired using inverse gated ${ }^{1} \mathrm{H}$ decoupling with the following parameters: sweep width of $201 \mathrm{ppm}, 3500$ scans, acquisition time of $0.3 \mathrm{~s}$, and a delay between scans of $4 \mathrm{~s}$, resulting in a total measurement time of $4 \mathrm{~h}$ and $10 \mathrm{~min}$. NMR tubes were spun at $20 \mathrm{~Hz}$ in the spectrometer to ensure sample homogeneity and short $1 \mathrm{D}$ ${ }^{1} \mathrm{H}$ spectra were acquired prior to ${ }^{19} \mathrm{~F}$ measurements to ensure decent field homogeneity was achieved. Gain settings were kept the same for all measurements. Chemical shifts were ascertained for all components by spiking an untreated homogenate with the appropriate standards. Chemical shift referencing for ${ }^{19} \mathrm{~F}$ was $\mathrm{CCl}_{3} \mathrm{~F}$ as stated in the IUPAC recommendations (Harris et al., 2002). Processing parameters prior to Fourier transformation included a $15 \mathrm{~Hz}$ line broadening and a backwards linear prediction in order to eliminate the broad signal due to the ${ }^{19} \mathrm{~F}$ background from the probe (40 points were back-predicted).

\section{Data Analysis}

We integrated all observed fluorine peaks of gemcitabine and metabolites, using the Bruker TOPSPIN software. Two integrals were measured: one for the reference $2 \mathrm{~F} 2 \mathrm{dA}$ and the other encompassing gemcitabine and its metabolites. We normalized the integrals to the tissue wet weights in order to obtain total gemcitabine metabolite concentration in samples in $\mu \mathrm{g} / \mathrm{mg}$ tissue.
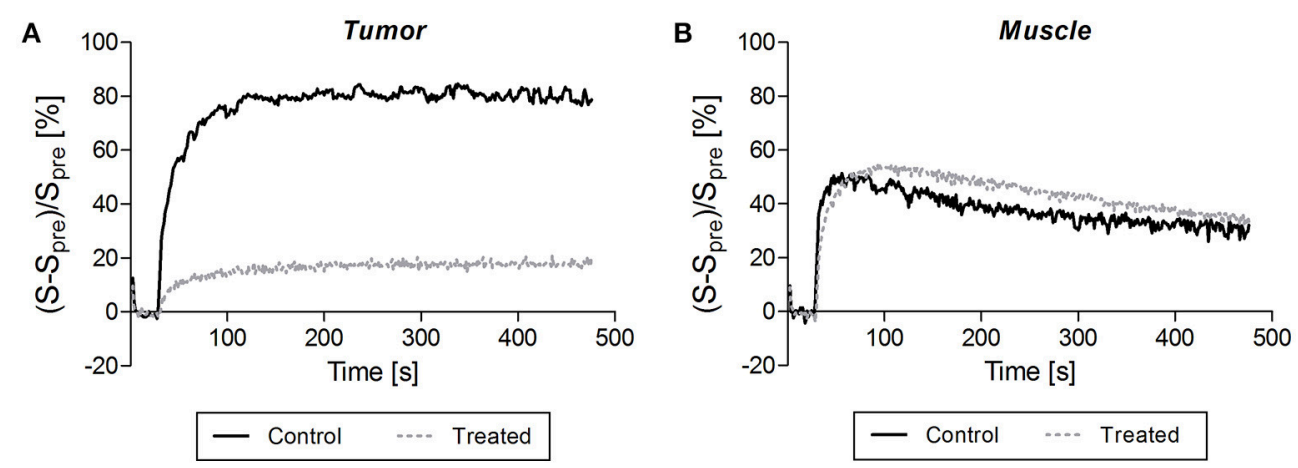

FIGURE 1 | Typical signal enhancement following contrast agent injection observed in tumor (A) and in muscle (B) of a control mouse (dark solid line) and a treated mouse (gray dotted line). (A) A smaller enhancement and a slower washout were typically observed in treated compared to untreated tumors. (B) By contrast, the enhancement pattern was similar in muscle of control and treated mice. 

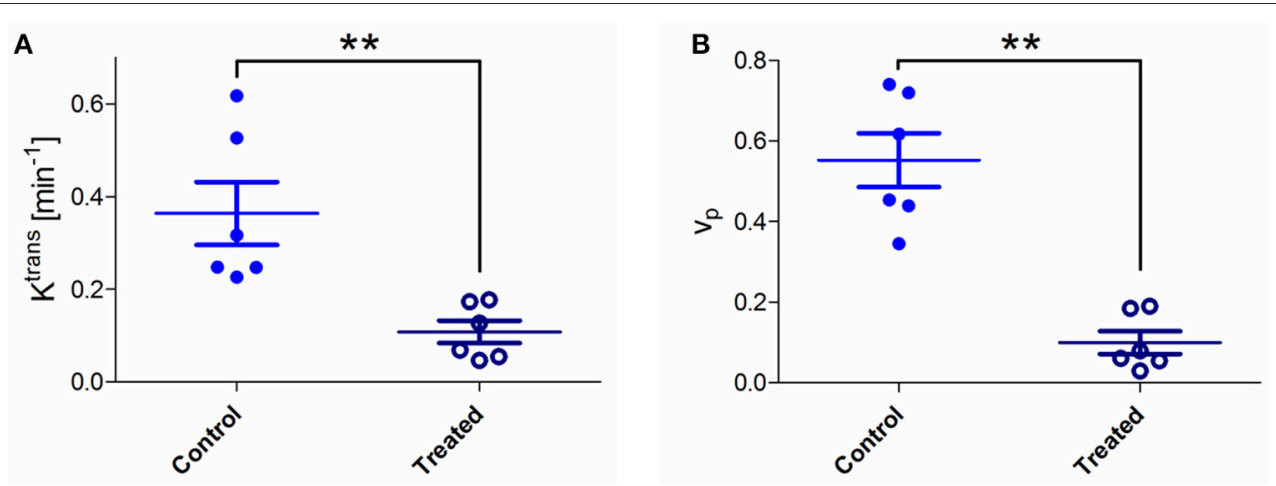

FIGURE 2 | (A) Absolute values of pharmacokinetic parameters $K^{\text {trans }}$ for control and treated tumors. A significant $K^{\text {trans }}$ decrease was observed in treated tumors compared to untreated ones (Mann-Whitney test; ${ }^{* *} p<0.01$ ). (B) Values of $V p$ (plasma volume fraction) for control and treated tumors. A significant decrease in $V p$ was observed in treated tumors compared to untreated ones (Mann-Whitney test; ${ }^{* \star} p<0.01$ ).

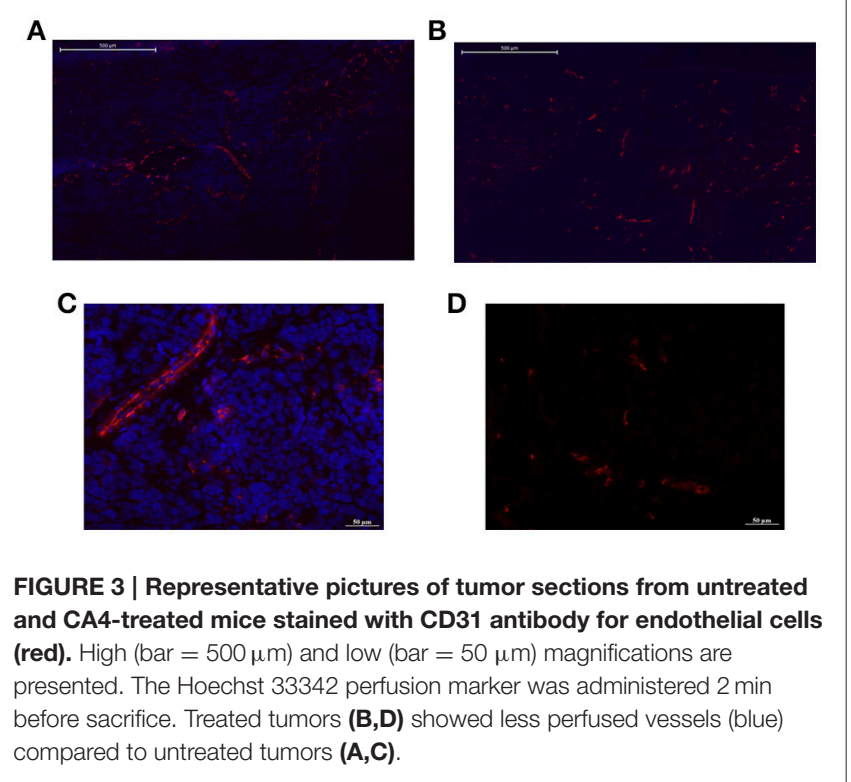

\section{Histology}

Six NMRI tumor-bearing mice were treated with vehicle $(n=$ $3)$ or CA4 $(n=3)$. Two hours after treatment, the functional perfusion marker Hoechst 33342 (15 mg/kg; iv injection; SigmaAldrich) was injected. Mice were sacrificed 2 min later. Five micrometer tumor cryosections were immunostained with a rat monoclonal antibody against CD31 (BD, clone MEC 13.3) and revealed with Alexa568 conjugated anti-rat secondary antibodies (Invitrogen). Tumor sections were imaged using a Zeiss Axioimager Z1 fluorescent microscope equipped with an Apotome module (Zeiss, Wetzlar, Germany).

Estimation of CA4-induced cell death was also obtained from TLT tumor-bearing mice treated with CA4 $(n=7)$ or vehicle $(n=8)$. Tumors were excised at day 1 after treatment initiation. Tumors were embedded in Tissue-Tek OCT compound and frozen in liquid nitrogen-cooled isopentane for cryosectioning. Samples were cut into $5 \mu \mathrm{m}$ sections. The frozen slices were stained with Haematoxylin \& Eosin or were probed for cellular death by TUNEL assay using an in situ cell death detection kit (Roche Diagnostics, Belgium). For TUNEL assay, nuclei were also counterstained with Hoechst 33342. Slides were then scanned with a Zeiss Mirax fluorescence microscope. Cell death was quantified using Frida software and expressed as a percentage of the whole tumor area.

\section{Statistical Analysis}

Values are presented as mean \pm standard error, unless otherwise stated. All statistical analysis was performed with GraphPad Prism 5. Non parametric Mann-Whitney tests were used to compare mean changes between control and treated groups. $P<$ 0.05 are considered statistically significant.

\section{RESULTS}

\section{CA4 Treatment Induced an Early Decrease in Tumor Blood Flow in TLT Tumors}

Figures 1, 2 illustrate DCE-MRI findings. DCE-MRI consists of the acquisition of T1-weighted images before, during, and after a contrast agent administration allowing for the generation of a signal intensity-time course from a tissue of interest. Figure 1 shows examples of enhancement curves (percentage of signal enhancement vs. time of selected region of interest) obtained from tumor (Figure 1A) and muscle (Figure 1B) of a control and a treated mouse. A smaller enhancement and a slower washout were typically observed in treated compared to untreated tumors (Figure 1A). By contrast, the enhancement pattern was similar in muscle of control and treated mice (Figure 1B).

The pharmacokinetic modeling of pixel signal intensitytime curve allowed extracting $\mathrm{K}^{\text {trans }}$ and $\mathrm{Vp}$ for each pixel. Mean tumor $\mathrm{K}^{\text {trans }}$ and $\mathrm{Vp}$ were calculated for each mouse by averaging all pixel values (Figure 2). A significant $K^{\text {trans }}$ decrease was observed in treated tumors compared to untreated tumors ( 0.11 vs. $0.36 \mathrm{~min}^{-1} ;{ }^{* *} p<0.01$; Figure $\left.2 \mathrm{~A}\right)$. $\mathrm{K}^{\text {trans }}$ values were lower in treated mice, particularly in tumor core regions. A 

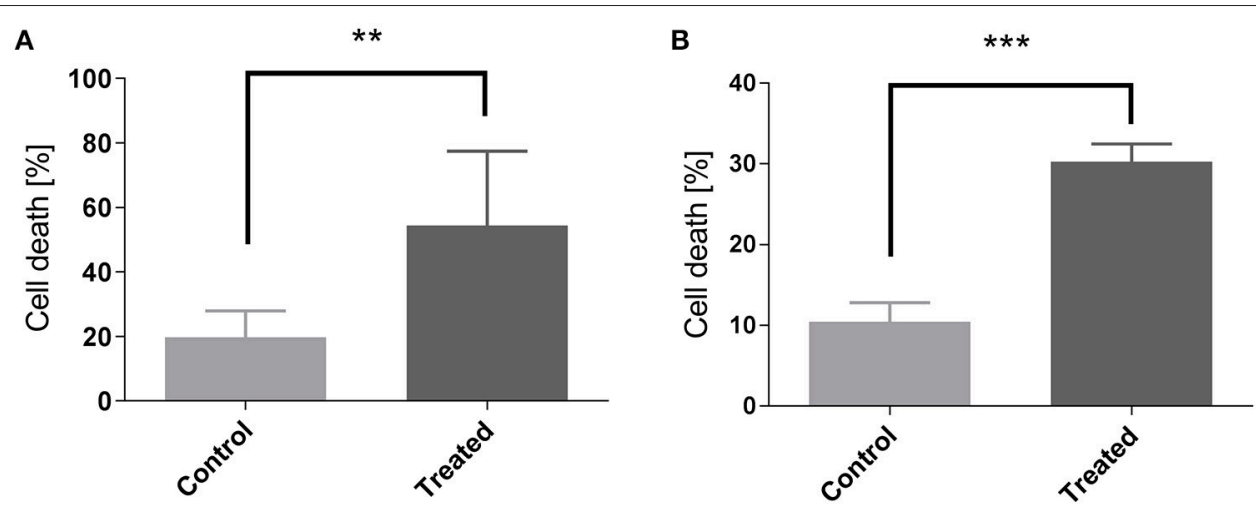

FIGURE 4 | Measurement of cellular death by quantification of Hematoxylin Eosine staining (A) and of TUNEL assay (B) in control and in CA4-treated tumors at day 1. Data are expressed as means \pm SEM (Unpaired $t$-tests; ${ }^{\star \star} p<0.01,{ }^{\star \star *} p<0.001$ ).
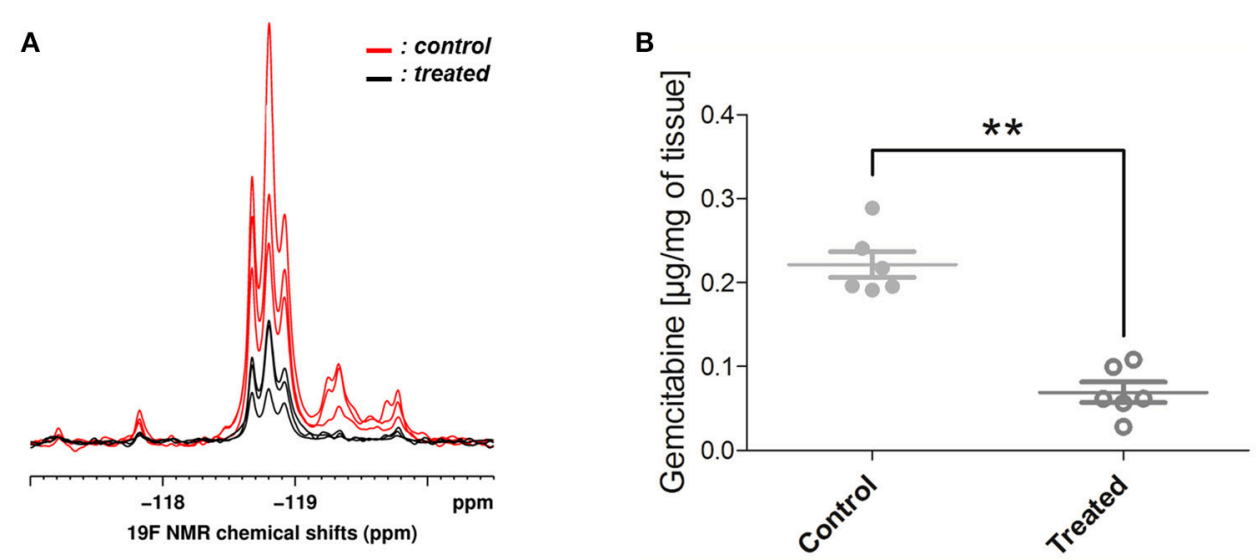

FIGURE 5 | ${ }^{19}$ F NMR findings. (A) Typical spectra obtained from 3 treated (in black) and 3 untreated (in red) tumor homogenates. Gemcitabine (dFdC) and two of its metabolites, $2^{\prime}, 2^{\prime}$-difluorodeoxyuridine ( $\mathrm{dFdU}$ ) and gemcitabine triphosphate (dFdCTP), were identified on the spectra. The metabolite dFdCTP was absent (or present in smaller quantities) in treated tumors. (B) Total gemcitabine uptake was quantified using an integral encompassing gemcitabine and its metabolites. A significant decrease of total gemcitabine uptake was observed in treated tumors when compared with untreated ones (Mann-Whitney test; ${ }^{* *} p<0.01$ ).

significant $\mathrm{Vp}$ decrease was also observed in treated tumors compared to untreated tumors $(0.099$ vs. $0.552 ; * * p<0.01)$ (Figure 2B).

Histology confirmed the blood flow shutdown seen by DCEMRI induced by CA4 treatment (Figure 3). Treated tumors (Figures 3A,C) showed less perfused vessels (assessed with Hoechst 33342, administered 2 min before sacrifice) compared to untreated tumors (Figures 3B,D). No difference was visually observed between groups in vascular density (assessed with CD31 antibody). We also observed an increase in tumor cell death 1 day after CA4 treatment. Histological analysis of Haematoxylin/Eosin -stained slices showed that tumors treated with CA4 present enlarged necrotic regions $(54 \pm 9 \%)$ as compared with tumors treated with vehicle $(20 \pm 3 \%)(* * p<$ 0.01 ) (Figure 4A). On the other hand, using the TUNEL assay (Figure 4B), positively stained areas were increased in treated tumors compared to control tumors ( 10 vs. $30 \%$; ${ }^{* * *} p<0.001$; Figure 4B).

\section{CA4 Treatment Induced a Decrease of Gemcitabine Uptake in TLT Tumors}

In ex vivo ${ }^{19} \mathrm{~F}$ NMR experiments, gemcitabine parent compound (dFdC) and two of its metabolites, 2',2'difluorodeoxyuridine $(\mathrm{dFdU})$ and gemcitabine triphosphate (2,2' -difluorodeoxycytidine-5' -triphosphate-dFdCTP) were identified. Typical spectra are shown on Figure 5A. We quantified the total gemcitabine amount using an integral encompassing gemcitabine and its metabolites. A significant decrease of total gemcitabine amount was observed in treated tumors when compared with untreated ones (0.07 vs. $0.22 \mu \mathrm{g} / \mathrm{mg}$ of tissue; ${ }^{* *} p<0.01$; Figure $\left.5 \mathbf{B}\right)$. Chemical shifts were determined by spiking an untreated tumor homogenate with appropriate standards. Results are shown on Figure 6. Respective assignments in ${ }^{19} \mathrm{~F}$ NMR spectra were $-54.6 \mathrm{ppm}$ for external standard $2 \mathrm{~F} 2 \mathrm{dA},-118.7 \mathrm{ppm}$ for $\mathrm{dFdC}$, two doublets at -118.2 and $-119.25 \mathrm{ppm}(J=241 \mathrm{~Hz}$; i.e., four frequency lines at $-117.8,-118.6,-118.8$, and $-119.7 \mathrm{ppm})$ 


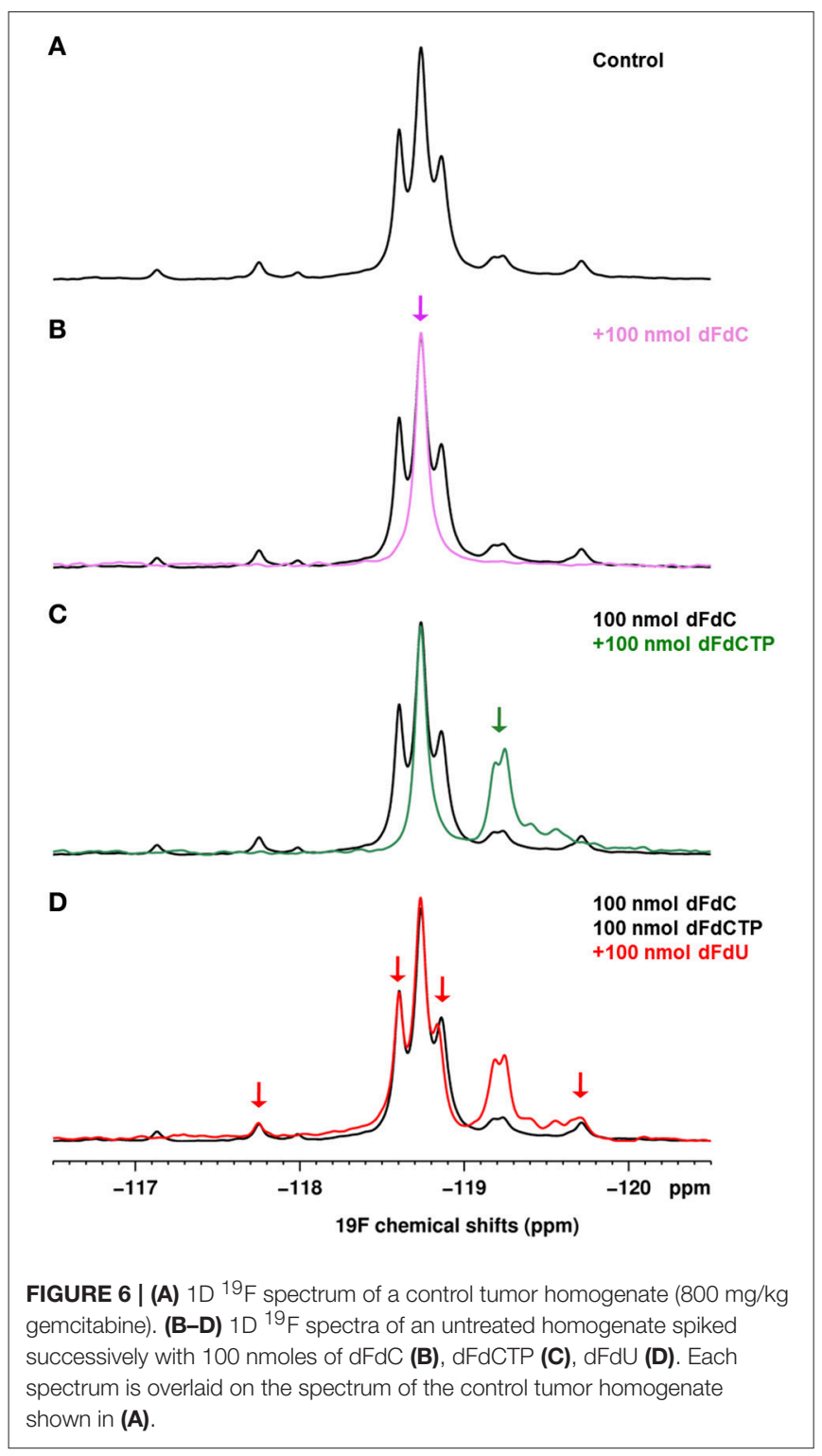

for dFdU, and a doublet at $-119.25 \operatorname{ppm}(J=15 \mathrm{~Hz})$ for dFdCTP. One can observe that the metabolite dFdCTP was present in smaller quantities in treated tumors compared to untreated tumors (Figure 5). For quality assurance purpose, we measured the area under the curve of the external standard $2 \mathrm{~F} 2 \mathrm{dA}$ for each sample and observed that it was stable from experiment to experiment, with a standard deviation of $<5 \%$ of the mean.

\section{DISCUSSION}

In this work, we demonstrate that a negative modulation of tumor blood flow by CA4 treatment causes a significant decrease in gemcitabine delivery in a solid tumor mouse model. It outlines the need to assess the impact of treatments on the tumor vascular function, for instance using DCE-MRI, when appraising a novel drug combination.
CA4 acts as a tubulin-binding agent, leading to a destabilization of the tubulin polymers of the cytoskeleton of proliferating endothelial cells. This way, an acute increase in tumor vascular permeability is induced, which in turn triggers several changes that together, decrease blood flow (Tozer et al., 2005). DCE-MRI confirmed the blood flow shutdown at $2 \mathrm{~h}$ after CA4 treatment, as revealed by reduced enhancement patterns as well as $\mathrm{K}^{\text {trans }}$ and $\mathrm{Vp}$ decrease in treated tumors, in comparison to untreated ones (Figure 2). The parameter $\mathrm{K}^{\text {trans }}$ represents the rate of transfer of the CtAg from the blood to the interstitial space. The physiological interpretation depends on the balance between capillary permeability and blood flow in the tumor: higher permeability makes $\mathrm{K}^{\text {trans }}$ more reflective of blood flow (flow-limited situation), whereas lower permeability makes $\mathrm{K}^{\text {trans }}$ more reflective of vascular permeability (permeability-limited situation; Tofts et al., 1999; O'Connor et al., 2007; Zweifel and Padhani, 2010). However, we previously reported that, in case of VDA assessment, $\mathrm{K}^{\text {trans }}$ changes determined with small molecular weight CtAg are more reflective of tumor blood flow changes, due to the high permeability of the CtAg across the capillary membrane (corresponding to a flow-limited situation; Fruytier et al., 2014b). Here, the observed $\mathrm{K}^{\text {trans }}$ decrease (Figure 2A) can thus be related to the early tumor blood flow shutdown induced by CA4, as confirmed by histology. The observed decrease in Vp (Figure 2B), the plasma volume fraction, is also consistent with the tumor blood flow shutdown. Of note, histological studies confirmed that CA4-treated tumors were less perfused compared to untreated tumors (Figure 3). As a consequence of this blood flow shutdown, we also observed that tumors treated with CA4 present more necrotic and apoptotic areas $24 \mathrm{~h}$ post-treatment (Figure 4).

In a second step, we investigated how the induced tumor blood flow shutdown can impact the delivery of chemotherapy, using ex vivo ${ }^{19} \mathrm{~F}$ NMR spectroscopy to determine the levels of gemcitabine and its major metabolites in tumors. Gemcitabine is a prodrug that must be phosphorylated intracellularly by deoxycytidine kinase to exhibit cytotoxic activities. Its major intracellular active metabolite is dFdCTP, although it remains in a constant ratio with gemcitabine monophosphate and diphosphate (Heinemann et al., 1992). Gemcitabine is inactivated mainly by deoxycytidine deaminase mediated conversion to dFdU (Mini et al., 2006). The ${ }^{19}$ F NMR experiments showed that the total gemcitabine uptake into the tumor was significantly lower in treated mice (CA4+gemcitabine) compared to controls (vehicle+gemcitabine) (Figure 5). These results are in line with those of Kristjansen et al. which demonstrated in ex vivo perfused human small cell lung cancer that the initial intratumor distribution of dFdC is flow dependent (Kristjansen et al., 1996). Gemcitabine, its three phosphorylated metabolites and dFdU overlap in ${ }^{19} \mathrm{~F}$ spectrum, making accurate quantification for individual metabolites difficult (Blackstock et al., 2001). However, it is clear that the intensity of the peak of the most active metabolite dFdCTP was lower in treated tumors compared to controls, or in some cases, null.

Several studies reported that blood flow reductions following VDA treatment could potentiate chemotherapy efficacy by trapping chemotherapy within the tumor and consequently 
increasing tumor exposure to the drug (Siim et al., 2003; Siemann, 2011). This has been observed for combinations where VDA was given after chemotherapy but also for VDA given before chemotherapy. For instance, Pruijn et al reported that the administration of the antivascular agent 5,6-dimethylxanthenone-4-acetic acid $2 \mathrm{~h}$ before the chemotherapeutic agent melphalan leads to an increase of the alkylating agent levels in the tumor and consequently to an enhanced activity (Pruijn et al., 1997). Additional studies reported that concurrent administration of VDA and chemotherapy increased antitumor activity (Morinaga et al., 2003; Kleespies et al., 2005).

However, as outlined in the present work, the induced blood flow shutdown could also decrease drastically the delivery of the chemotherapeutic agent inside the tumor. The potentiation of chemotherapy by drug trapping will take place only if the chemotherapeutic agent can reach the tumor before the vascular shutdown. Predictive biomarkers of drug delivery could thus be particularly useful in assessing novel drug combinations. For that purpose, we appraised the potential of DCE-MRI using low molecular weight CtAg which could mimic small conventional cytotoxic drugs in such a way that their delivery inside the tumor could be reflective of uptake and distribution of conventional chemotherapy (Artemov et al., 2001). In addition to be a pharmacodynamic biomarker of CA4 treatment, the DCE-MRI parameter $\mathrm{K}^{\text {trans }}$ was predictive of the gemcitabine delivery. We observed a similar decrease of $\mathrm{K}^{\text {trans }}$ and gemcitabine delivery in the parallel groups of mice (70 and $68.7 \%$ of decrease, respectively).

Response to chemotherapy is determined by the delivery of cytotoxic agent to the tumor but also by chemotherapy cellular uptake, retention, and metabolic activation, as well as by chemotherapy sensitivity in the target tumor cells (van Laarhoven et al., 2007; Jain, 2012). Moreover, in addition to any spatial cooperation between both treatments, final outcome following treatment combination will be also affected by the effect of the VDA on the tumor (cell/vasculature; Wang et al., 2012). The microenvironmental changes secondary to VDA-induced blood flow shutdown can also modulate the sensitivity of chemotherapy (Pruijn et al., 1997; Siim et al., 2003). Indeed, on the one hand,

\section{REFERENCES}

Artemov, D., Solaiyappan, M., Bhujwalla, Z. M. (2001). Magnetic resonance pharmacoangiography to detect and predict chemotherapy delivery to solid tumors. Cancer Res. 61, 3039-3044.

Bapiro, T. E., Richards, F. M., Goldgraben, M. A., Olive, K. P., Madhu, B., Frese, K. K., et al. (2011). A novel method for quantification of gemcitabine and its metabolites $2^{\prime}, 2^{\prime}$-difluorodeoxyuridine and gemcitabine triphosphate in tumour tissue by LC-MS/MS: comparison with (19)F NMR spectroscopy. Cancer Chemother Pharmacol. 68, 1243-1253. doi: 10.1007/s00280-011-1613-0

Blackstock, A. W., Lightfoot, H., Case, L. D., Tepper, J. E., Mukherji, S. K., Mitchell, B. S., et al. (2001). Tumor uptake and elimination of $2^{\prime}, 2^{\prime}$-difluoro$2^{\prime}$-deoxycytidine (gemcitabine) after deoxycytidine kinase gene transfer: correlation with in vivo tumor response. Clin. Cancer Res. 7, 3263-3268.

Blakey, D. C., Westwood, F. R., Walker, M., Hughes, G. D., Davis, P. D., Ashton, S. E., et al. (2002). Antitumor activity of the novel vascular targeting agent ZD6126 in a panel of tumor models. Clin. Cancer Res. 8, 1974-1983. hypoxia and acidosis could be favorable to the activity of specific agents (e.g., bioreductive drugs, melphalan; Pruijn et al., 1997; Taraboletti et al., 2010), whereas, the severe glucose and oxygen deprivation induced by CA4P have been shown to induce GRP78 expression that could lead to drug resistance (Dong et al., 2005).

Our study could be considered as a proof-of-concept that directly links the effect of VDA on the blood flow to the delivery another chemotherapeutic agent. A limitation of our study is that we analyzed this effect only at one timing $(2 \mathrm{~h})$ post-VDA treatment. For the future, it could be interesting to follow the dynamics of this effect at different timings and to study the impact of the combination at different timings on the tumor response to treatments.

In conclusion, although the link between drug delivery and therapeutic efficiency is not straightforward, the present study demonstrates that the potential impact of VDA on intratumoral drug delivery should be taken into account when designing novel drug combination. For that purpose, the assessment of tumor vascular function by DCE-MRI could be helpful to avoid misinterpretation when a failure of treatment is observed, but also to maximize therapeutic gain.

\section{AUTHOR CONTRIBUTIONS}

AF conceived and designed the work, acquired, analyzed and interpreted data, drafted and revised the manuscript, approved the final version. $\mathrm{CL}, \mathrm{CP}, \mathrm{JM}, \mathrm{CB}$, and $\mathrm{MN}$ acquired, analyzed and interpreted data, revised the manuscript, approved the final version. OF and BJ analyzed and interpreted data, revised the manuscript, approved the final version. BG conceived and designed the work, analyzed and interpreted data, drafted and revised the manuscript, approved the final version. All authors agree to be accountable for all aspects of the work.

\section{ACKNOWLEDGMENTS}

This study was supported by a grant from the Belgian Foundation against cancer, ARC 09/14-020, the Fonds Joseph Maisin, and the Fonds du patrimoine. This study is a part of the Ph.D. thesis of Anne-Catherine Fuytier (Fruytier, 2014).

Buckley, D. L., and Parker, G. J. M. (2005). "Measuring contrast agent concentration in T1-weighted dynamic contrast-enhanced, MRI," in Dynamic Contrast-Enhanced Magnetic Resonance Imaging in Oncology, eds A. Jackson, D. L. Buckley, and G. J. M. Parker (Berlin: Springer), 69-79. doi: 10.1007/3-540-26420-5_5

Cesca, M., Bizzaro, F., Zucchetti, M., and Giavazzi, R. (2013). Tumor delivery of chemotherapy combined with inhibitors of angiogenesis and vascular targeting agents. Front Oncol. 3:259. doi: 10.3389/fonc.2013.00259

Chaplin, D. J., and Hill, S. A. (2002). The development of combretastatin A4 phosphate as a vascular targeting agent. Int. J. Radiat. Oncol. Biol. Phys. 54, 1491-1496. doi: 10.1016/S0360-3016(02)03924-X

Dong, D., Ko, B., Baumeister, P., Swenson, S., Costa, F., Markland, F., et al. (2005). Vascular targeting and antiangiogenesis agents induce drug resistance effector GRP78 within the tumor microenvironment. Cancer Res. 65, 5785-5791. doi: 10.1158/0008-5472.CAN-05-0754

Folkman, J. (1995). Angiogenesis in cancer, vascular, rheumatoid and other disease. Nat. Med. 1, 27-31. doi: 10.1038/nm0195-27 
Fruytier, A. C. (2014). Evaluation of Tumor Hemodynamics by Magnetic Resonance Imaging: Consequence of a Modulation of Perfusion on Chemotherapy Delivery. Ph.D. thesis, Université catholique de Louvain, Brussels.

Fruytier, A. C., Magat, J., Colliez, F., Jordan, B., Cron, G., Gallez, B. (2014a). Dynamic contrast-enhanced MRI in mice at high field: estimation of the arterial input function can be achieved by phase imaging. Magn. Reson. Med. 71, 544-550. doi: 10.1002/mrm.24682

Fruytier, A. C., Magat, J., Neveu, M. A., Karroum, O., Bouzin, C., Feron, O., et al. (2014b). Dynamic contrast-enhanced MRI in mouse tumors at 11.7T: Comparison of 3 contrast agents with different molecular weights to assess the early effects of combretastatin A4. NMR Biomed. 27, 1403-1412. doi: $10.1002 / \mathrm{nbm} .3220$

Grosios, K., Holwell, S. E., McGown, A. T., Pettit, G. R., and Bibby, M. C. (1999). In vivo and in vitro evaluation of combretastatin A-4 and its sodium phosphate prodrug. Br. J. Cancer 81, 1318-1327. doi: 10.1038/sj.bjc.6692174

Harris, R. K., Becker, E. D., Cabral de Menezes, S. M., Goodfellow, R., and Granger, P. (2002). NMR Nomenclature: nuclear spin properties and conventions for chemical shifts. IUPAC Recommendations 2001. Solid State Nucl. Magn. Reson. 22, 458-483. doi: 10.1006/snmr.2002.0063

Heinemann, V., Xu, Y. Z., Chubb, S., Sen, A., Hertel, L. W., Grindey, G. B., et al. (1992). Cellular elimination of $2^{\prime}, 2^{\prime}$-difluorodeoxycytidine $5^{\prime}$-triphosphate: a mechanism of self-potentiation. Cancer Res. 52, 533-539.

Jain, R. K. (2012). Delivery of molecular and cellular medicine to solid tumors. Adv. Drug Deliv. Rev. 64(Suppl.), 353-365. doi: 10.1016/j.addr.2012. 09.011

Kleespies, A., Köhl, G., Friedrich, M., Ryan, A. J., Barge, A., Jauch, K. W., et al. (2005). Vascular targeting in pancreatic cancer: the novel tubulin-binding agent ZD6126 reveals antitumor activity in primary and metastatic tumor models. Neoplasia 7, 957-966. doi: 10.1593/neo.05304

Kristjansen, P. E., Brown, T. J., Shipley, L. A., and Jain, R. K. (1996). Intratumor pharmacokinetics, flow resistance, and metabolism during gemcitabine infusion in ex vivo perfused human small cell lung cancer. Clin. Cancer Res. 2, 359-267.

Maxwell, R. J., Wilson, J., Prise, V. E., Vojnovic, B., Rustin, G. J., Lodge, M. A., et al. (2002). Evaluation of the anti-vascular effects of combretastatin in rodent tumours by dynamic contrast enhanced MRI. NMR Biomed. 15, 89-98. doi: $10.1002 / \mathrm{nbm} .754$

Mini, E., Nobili, S., Caciagli, B., Landini, I., and Mazzei, T. (2006). Cellular pharmacology of gemcitabine. Ann. Oncol. 17(Suppl. 5), v7-12. doi: 10.1093/annonc/mdj941

Morinaga, Y., Suga, Y., Ehara, S., Harada, K., Nihei, Y., and Suzuki, M. (2003). Combination effect of AC-7700, a novel combretastatin A-4 derivative, and cisplatin against murine and human tumors in vivo. Cancer Sci. 94, 200-204. doi: 10.1111/j.1349-7006.2003.tb01419.x

Nielsen, T., Murata, R., Maxwell, R. J., Stødkilde-Jørgensen, H., Øtergaard, L., Ley, C. D., et al. (2010). Non-invasive imaging of combretastatin activity in two tumor models: association with invasive estimates. Acta Oncol. 49, 906-913. doi: 10.3109/0284186X.2010.499135

O'Connor, J. P. B., Jackson, A., Parker, G. J. M., and Jayson, G. C. (2007). DCE-MRI biomarkers in the clinical evaluation of antiangiogenic and vascular disrupting agents. Br. J. Cancer 96, 189-195. doi: 10.1038/sj.bjc.6603515

O’Connor, J. P., Jackson, A., Parker, G. J., Roberts, C., and Jayson, G. C. (2012). Dynamic contrast-enhanced MRI in clinical trials of antivascular therapies. Nat. Rev. Clin. Oncol. 9, 167-177. doi: 10.1038/nrclinonc.2012.2

Olive, K. P., Jacobetz, M. A., Davidson, C. J., Gopinathan, A., McIntyre, D., Honess, D., et al. (2009). Inhibition of Hedgehog signaling enhances delivery of chemotherapy in a mouse model of pancreatic cancer. Science 324, 1457-1461. doi: $10.1126 /$ science. 1171362
Pruijn, F. B., van Daalen, M., Holford, N. H., and Wilson, W. R. (1997). Mechanisms of enhancement of the antitumour activity of melphalan by the tumour-blood-flow inhibitor 5,6-dimethylxanthenone-4-acetic acid. Cancer Chemother. Pharmacol. 39, 541-546. doi: 10.1007/s002800050611

Ratner, A. V., Quay, S., Muller, H. H., Simpson, B. B., Hurd, R., and Young, S. W. (1989). 19F relaxation rate enhancement and frequency shift with Gd-DTPA. Invest. Radiol. 24, 224-247. doi: 10.1097/00004424-198903000-00010

Siemann, D. W. (2011). The unique characteristics of tumor vasculature and preclinical evidence for its selective disruption by Tumor-Vascular Disrupting Agents. Cancer Treat. Rev. 37, 63-74. doi: 10.1016/j.ctrv.2010.05.001

Siim, B. G., Lee, A. E., Shalal-Zwain, S., Pruijn, F. B., McKeage, M. J., and Wilson, W. R. (2003). Marked potentiation of the antitumour activity of chemotherapeutic drugs by the antivascular agent 5,6-dimethylxanthenone4-acetic acid (DMXAA). Cancer Chemother Pharmacol. 51, 43-52. doi: 10.1007/s00280-002-0529-0

Taper, H. S., Woolley, G. W., Teller, M. N., and Lardis, M. P. (1966). A new transplantable mouse liver tumor of spontaneous origin. Cancer Res. 26, 143-148.

Taraboletti, G., Bonezzi, K., and Giavazzi, R. (2010). "Combination therapy with chemotherapy and VDAs," in Vascular Disruptive Agents for the Treatment of Cancer, ed T. Meyer (New York, NY: Springer), 77-93. doi: 10.1007/978-1-4419-6609-4_4

Thorpe, P. E. (2004). Vascular targeting agents as cancer therapeutics. Clin. Cancer Res. 10, 415-427. doi: 10.1158/1078-0432.CCR-0642-03

Tofts, P. S., Brix, G., Buckley, D. L., Evelhoch, J. L., Henderson, E., Knopp, M. V., et al. (1999). Estimating kinetic parameters from dynamic contrast-enhanced T(1)-weighted MRI of a diffusable tracer: standardized quantities and symbols. J. Magn. Reson. Imaging 10, 223-232. doi: 10.1002/(SICI)1522-2586(199909)10:3 <223::AID-JMRI2>3.0.CO;2-S

Tozer, G. M., Kanthou, C., and Baguley, B. C. (2005). Disrupting tumour blood vessels. Nat. Rev. Cancer 5, 423-435. doi: 10.1038/nrc1628

Tozer, G. M., Kanthou, C., and Chaplin, D. J. (2008). "Vascular disrupting agents in cancer therapy," in Tumor Angiogenesis, eds D. Marmé and N. Fusenig (Berlin: Springer), 809-829.

van Laarhoven, H. W., Klomp, D. W., Rijpkema, M., Kamm, Y. L., Wagener, D. J., Barentsz, J. O., et al. (2007). Prediction of chemotherapeutic response of colorectal liver metastases with dynamic gadolinium-DTPA-enhanced MRI and localized 19F MRS pharmacokinetic studies of 5-fluorouracil. NMR Biomed. 20, 128-140. doi: 10.1002/nbm.1098

Wang, E., Pili, R., and Seshadri, M. (2012). Modulation of chemotherapeutic efficacy by vascular disrupting agents: optimizing the sequence and schedule. J. Clin. Oncol. 30, 760-761. doi: 10.1200/JCO.2011.39.3934

Zweifel, M., and Padhani, A. R. (2010). Perfusion MRI in the early clinical development of antivascular drugs: decorations or decision making tools? Eur. J. Nucl. Med. Mol. Imaging 37(Suppl. 1), S164-S182. doi: $10.1007 / \mathrm{s} 00259-010-1451-\mathrm{z}$

Conflict of Interest Statement: The authors declare that the research was conducted in the absence of any commercial or financial relationships that could be construed as a potential conflict of interest.

Copyright (c) 2016 Fruytier, Le Duff, Po, Magat, Bouzin, Neveu, Feron, Jordan and Gallez. This is an open-access article distributed under the terms of the Creative Commons Attribution License (CC BY). The use, distribution or reproduction in other forums is permitted, provided the original author(s) or licensor are credited and that the original publication in this journal is cited, in accordance with accepted academic practice. No use, distribution or reproduction is permitted which does not comply with these terms. 